\title{
Sepsis and tachycardia: Etiologic factors and effects on prognosis
}

\author{
Baygin O, MD, Kararmaz A, MD
}

Department of Anesthesiology, Marmara University School of Medicine, Istanbul, Turkey

Background\&Aims:

Despite advances in medical science, especially researches have focused on early detection and treatment of sepsis, it is still a serious medical condition triggered by infection, characterized by uncontrollable systemic inflammatory response that frequently leading to mortality and morbidity in intensive care unit.

Patients with sepsis predispose cardiac arrhythmias because of excessive inflammation and circulating stress hormone.

The present study aimed to identify etiologic factors of tachycardia in critically ill patients with sepsis. We also investigated effects of tachycardia on prognosis.

Materials and Methods: Following the approval of the Marmara University School of Medicine Ethic Committee and written informed consent of patients' relatives, a prospective observational study was conducted in adult patients who were diagnosed with sepsis (according to American College of Chest Physicians/Society of Critical Care Medicine Consensus Conference Committee) and followed over than 48 hours in intensive care unit. Exclusion criterias were pregnancy, severe valvular diseases and coronary artery disease, extracorporeal membrane oxygenation usage and patients who does not need mechanical ventilatory support. Demographic data, comorbid diseases, and clinical and laboratory data were collected prospectively. Patients were followed up until death or hospital discharge. Early goal-directed therapy used to achieve hemodynamic optimization. The required sample size was estimated by a software package (G-Power@ 3.0.10 PC; by Fraz Faul, Universtat Kiel, Kiel, Germany) and calculated to 50 (Correlation coefficient $(\rho)$ is 0.4 and above, type I error probability $(\alpha)$ is 0.05 and $90 \%$ power, $\alpha 0.05$, power 0.9 ). Statistical analysis was performed using Statistical Package for Social Sciences for Windows version 21.0 (SPSS Inc., Chicago, IL, USA). Continuous variables are presented as mean \pm standard deviation (SD). The KolmogorovSmirnov test was used to assess the data normality.

Results: Thirty men and twenty women, a total of $\mathbf{5 0}$ patients were included to study. We observed tachycardia in all patients during follow up. The time of heart rate greater than $100 \mathrm{bpm}$ was $34.6 \pm 39.7$ (\%95 Cl 23.3-45.9). There was a statistically significant correlation between tachycardia and $\mathrm{pH}$, mean arterial pressure (MAP), base excess, temperature, and procalcitonin level $(p<0.05)$. When these factors were analyzed with linear regression, it was found that MAP, temperature, and procalcitonin levels were associated with increased heart rate $(p<0.05)$. Eighteen patients were discharged from intensive care unit and 32 patients died. Duration of tachycardia, APACHE and SOFA scores were associated with mortality.

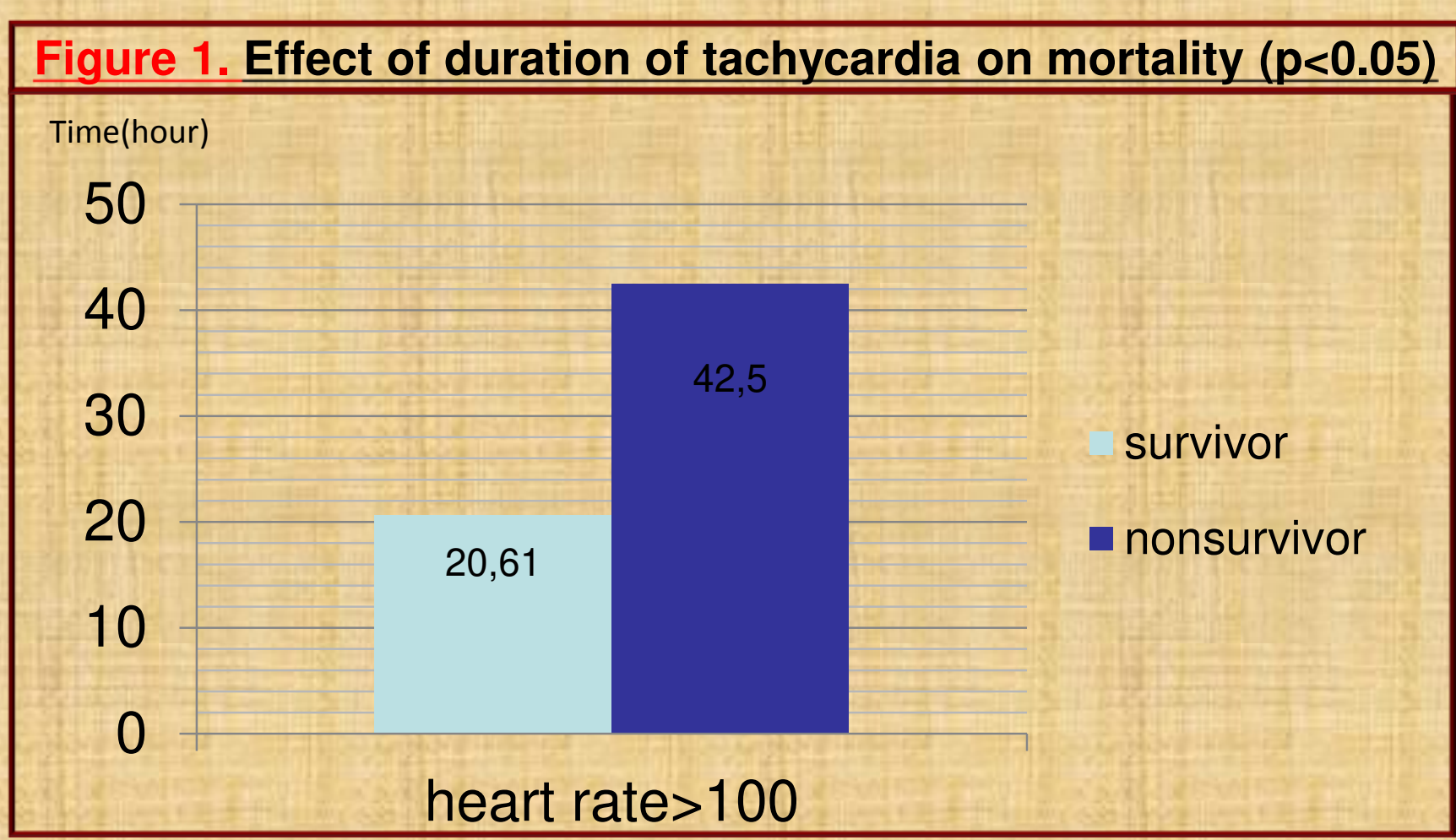

Table 2. Linear regression analysis of etiologic factors of tachycardia

Coefficients* \begin{tabular}{|l|l|l|l|}
\hline Model & $\begin{array}{l}\text { Unstandardized } \\
\text { Coefficients }\end{array}$ & $\begin{array}{l}\text { Standardized } \\
\text { Coefficients }\end{array}$ & Sig.
\end{tabular}

B Std. Beta

\begin{tabular}{|lrl|l|l|} 
& & Error & & \\
(Constant) & 269.138 & 260.149 & 1.035 & 0.307
\end{tabular}

$\begin{array}{llllll}\text { Mean arterial }-0.147 & 0.072 & -0.238 & -2.027 & 0.049\end{array}$

pressure

\begin{tabular}{lllllll|} 
Hemoglobin & -0.808 & 0.725 & -0.133 & -1.115 & 0.271 \\
\hline pH & -40.345 & 33.588 & -0.271 & -1.201 & 0.236 \\
\hline pco2 & -0.020 & 0.220 & -0.016 & -0.092 & 0.927 \\
\hline Base excess & -0.179 & 0.497 & -0.077 & -0.360 & 0.721 \\
\hline $\begin{array}{l}\text { Body } \\
\text { temperature }\end{array}$ & 4.199 & 1.742 & 0.271 & 2.410 & 0.020 \\
$\begin{array}{l}\text { Procalcitonin } \\
0.120\end{array}$ & 0.052 & 0.274 & 2.323 & 0.025
\end{tabular}

Conclusion: The incidence of tachycardia is very high in cases followed up with sepsis in intensive care unit. According to our findings, low mean arterial pressure, high body temperature and high procalcitonin values were important factors in tachycardia etiology. The relationship between heart rate and arterial blood pressure and body temperature is already well known. But this study showed that there may be also a relationship between inflammation and tachycardia. New studies are needed to investigate changes in heart rate during different periods of inflammation (proinflammatory/anti-inflammatory). This study also showed that duration of tachycardia may be related with mortality. 